\title{
Neoliberalismo y territorio en la Argentina de fin de siglo
}

\author{
Mabel Manzanal ${ }^{1}$ \\ CONICET/Universidad de Buenos \\ Aires
}

\section{Introducción}

En Argentina, el tema de las economías regionales se desarrolla originalmente en torno a la cuestión de las desigualdades regionales, que se manifiestan entre la región pampeana (privilegiada en la política, en las finanzas, en las comunicaciones, en la concentración del capital, en el dominio de la tecnología y en el nivel de capacitación e investigación) y el resto del país (con manifestaciones de mayor subdesarrollo y marginalidad social y económica).

Hoy día estas desigualdades siguen vigentes, persisten y se han agravado; lo cual no siempre se puede corroborar fehacientemente a partir de las estadísticas disponibles en el ámbito de cada provincia.

En Argentina existe, históricamente, una carencia importante de producción de información básica confiable y comparable entre distintas jurisdicciones y periodos, por diferentes razones, entre otras por el manejo político de la misma. Esta dificultad se acentuó desde los años noventa, con la aplicación rigurosa de la política neoliberal de ajuste macroeconómico, que se tradujo en el deterioro y/o desmantelamiento de muchos organismos encargados de la recopilación y sistematización de estadísticas en general. 


\section{Antecedentes}

Un primer referente que da cuenta de las desigualdades regionales es el producto bruto provincial por habitante (PBG/cápita). De todos modos, antes de adentrarnos en su comprensión, cabe advertir que el $\mathrm{PBG} /$ cápita no es el mejor indicador para evaluar las desigualdades regionales (aunque también es cierto que no existe otro para expresar en síntesis esta realidad). ${ }^{2}$

Una limitante del PBG en Argentina es que ha perdido su calidad y confiabilidad desde la desregulación neoliberal aplicada a partir del ajuste macroeconómico. El PBG era, y es, una serie anual elaborada por el Consejo Federal de Inversiones (CFI). Pero la reestructuración del CFI ha llevado a que dicha serie no se continúe hasta el presente en todas las provincias, y adolezca de problemas de comparabilidad entre las distintas jurisdicciones (por diferencias en el método de recopilación y sistematización aplicado). ${ }^{3}$

A pesar de las restricciones señaladas, utilizaremos el $\mathrm{PBG} /$ cápita porque las falencias metodológicas mencionadas no invalidan la observación del proceso de conformación inequitativa territorial de Argentina en sus aspectos más generales. La magnitud de la diferencia entre jurisdicciones es tan pronunciada, según puede verse en el cuadro 1 y en la gráfica 1, que también resulta una elocuente y confiable representación de lo que sucede en la realidad regional argentina (por otra parte, esta información se corrobora con otra complementaria que adjuntamos y

${ }^{2}$ Uno de los problemas de que adolece el PBG/cápita es que no refleja la profundidad de las desigualdades socio-territoriales, en tanto es un promedio influido por los altos niveles de ingresos de la población del estrato superior, lo cual se acentúa en países fuertemente polarizados en su estructura social, como sucede con los latinoamericanos. Así mismo, no refleja adecuadamente la realidad en ámbitos con escasa población y enormes ingresos públicos (como sucede en zonas con recursos provenientes de regalías de la explotación petrolífera o con otros beneficios especiales). En estos casos no expresa la situación socioeconómica de la mayoría de la población local (lo que sucede en las provincias del sur argentino, productoras de gas y petróleo, o en la más austral de todas, Tierra del Fuego, con enormes ventajas vinculadas a la política pública de promoción industrial).

${ }^{3}$ La reestructuración del CFI implicó que las provincias dejaran de recibir asesoramiento (sistemático, generalizado y coordinado por el CFI para la confección de su PBG), quedando bajo la responsabilidad de cada una solicitar, o no, asistencia al respecto. Por ello, desde 1990 menguó la calidad del dato de PBG. Y no es factible reemplazarlo por el producto bruto interno - $\mathrm{PBI}$ - (equivalente del $\mathrm{PBG}$ pero en el ámbito nacional) a cargo del Ministerio de Economía, Obras y Servicios Públicos (MEOysP). Porque el PBI está elaborado sólo para el total nacional (por provincia sólo se cuenta con la serie del año 1993). 
con lo que es conocido de la observación, de la experiencia y del conocimiento de la historia de los procesos socioeconómicos).

$\mathrm{Al}$ analizar el $\mathrm{PBG} /$ cápita de 1996 para las 24 jurisdicciones (23 provincias más la Capital Federal) se destaca: (i) el amplio rango de disparidad entre los valores máximos y mínimos: de \$1 mil 978 en Santiago del Estero llega a \$21 970 en la Ciudad de Buenos Aires; y (ii) la conformación de dos conjuntos de provincias diferenciadas según sus montos de ingresos por habitante: uno formado por las del norte, noreste y noroeste con menos de $\$ 5$ mil por habitante, y el otro por las pampeanas y patagónicas que superan los $\$ 7000$ por habitante -llegando al de Capital Federal a un máximo superior a los $\$ 20000$ por habitante.

De todos modos, algunos casos merecen explicaciones particulares, por las restricciones arriba mencionadas. Nos referimos especialmente al de Mendoza. Ésta es en realidad una provincia integrante del área desarrollada del país, sin embargo figura en estos datos en el grupo de menor porcentaje de $\mathrm{PBG} /$ habitante. Ello se debe a un problema de comparabilidad de la información, pues en ella se aplicaron métodos y criterios de cálculo del PBG independientes de la coordinación centralizada del CFI, precisamente por las razones aducidas arriba. Por su parte, las provincias patagónicas (Santa Cruz, Tierra del Fuego, Chubut, Neuquén y Río Negro) están en el mismo grupo que las pampeanas. Y ello se debe, como señalamos, a su escasa población y sus altos ingresos provenientes de las regalías petrolíferas. La Rioja es un caso especial, pues por ser la provincia natal del presidente de la nación, ha recibido durante el periodo correspondiente beneficios especiales (y además tampoco tiene mucha población).

En el mismo cuadro figuran los indicadores de necesidades básicas insatisfechas (NBI) para las 24 jurisdicciones. Esta serie corrobora lo ya señalado con las anteriores. Obsérvese el elevado rango de separación entre la Capital Federal y Formosa; quedando en la gráfica 1 mejor evidenciadas las desigualdades entre las distintas jurisdicciones. Nótese el comportamiento opuesto pero complementario del PBG/cápita y de las NBI: a medida que disminuye el $\mathrm{PBG} /$ habitante aumentan los indicadores de pobreza (las jurisdicciones que tienen menor ingreso por habitante son las que también tienen mayores índices de pobreza en su población).

Otra información que muestra lo mismo es el Índice de Desarrollo Humano (IDH), aunque modera las diferencias entre jurisdicciones. Esto ocurre porque en su construcción, además del ingreso per cápita, se incorporan otras variables, en general 
de carácter estructural, de largo plazo (que suelen aminorar las diferencias entre zonas): esperanza de vida al nacer, privación de longevidad, tasa de analfabetismo, privación de analfabetismo, tasa de escolarización, privación de escolarización, privación de educación, utilidad del ingreso, privación de desarrollo humano. En el cuadro 2 la ciudad de Buenos Aires detenta, de nuevo, la mejor situación, y se mantiene el orden entre las distintas provincias en general, con algunas excepciones (en especial Mendoza, que pasa a estar entre las provincias con mejor IDH, tal como advertimos). Nótese que el IDH varía entre 0 y 1 (siendo 1 la referencia que indica la mejor situación en $\mathrm{DH}$ ) y la variación entre los extremos, Ciudad de Buenos Aires y Formosa, es de un 20 por ciento.

Con todo, estas evidencias no indican que la cuestión regional mantenga las características distintivas de la década de los setenta o de los ochenta, porque la singularidad del problema territorial ha sido notablemente modificada a partir de los ochenta. La organización territorial ha experimentado cambios radicales, no siempre perceptibles, que resultan del nuevo escenario tecnológico, informático, socioeconómico y político, y que en el transcurso de las próximas décadas, seguramente, se profundizarán.

Análisis en el ámbito del primer mundo apuntan a la "competitividad sistémica" como el desafío que deben encarar las localidades o regiones que busquen "crecimiento económico con desarrollo social" (o con mejor distribución de sus resultados). Estos estudios proponen instalar, de modo local o regional, formas competitivas que alcancen al conjunto, o a la mayor parte de los sectores productivos y sociales de la comunidad o zona en cuestión, y que conformen mallas de vinculación (económica, productiva, social, cultural) entre empresarios, proveedores, consumidores, municipios, instituciones científicas, civiles, públicas, y población comunitaria. Se trata de una articulación dirigida a conformar un espacio intangible de flujos en constante evolución, una nueva morfología socio-territorial con su respectiva lógica espacial (Caravaca, 1998:3).

La pregunta que desde el análisis y desde la praxis de la realidad argentina se nos plantea, es: ¿existe en las áreas extrapampeanas viabilidad para este tipo de políticas? Y ¿son ellas potencialmente transformadoras de la grave polarización social y económica del presente?

Nuestro marco conceptual de análisis de los procesos macroeconómicos y sus efectos territoriales parte de la premisa (re- 
petidamente corroborada) de que la historia del desarrollo de las regiones en Argentina, el devenir productivo y económico del interior extrapampeano no ha conducido al desarrollo. Por el contrario, su particularidad es, en términos de Notcheff (1996:113 y ss.), una "sucesión de burbujas sin desarrollo", la mudanza entre crecimiento y estancamiento. Se trata de etapas de expansión o crecimiento, "burbujas", sucedidas por otras de estancamiento. Todo lo contrario al "desarrollo", proceso impulsado por innovaciones, por cuasi rentas tecnológicas generadas por las empresas y por los sistemas nacionales de innovación, constituyendo en tal sentido una "opción dura" para la élite empresaria nacional (ibid.).

En otras palabras, lo que ha sucedido en Argentina, y en sus economías regionales, es el aprovechamiento de las opciones "blandas", sin innovación tecnológica, ni acumulación, ni inversión de capital, necesarios para consolidar todo proceso de desarrollo. La expansión o el crecimiento regional cuando se produjo fue transitorio, siguiéndole periodos de estancamiento y crisis. Y la explicación de este proceso está en que la expansión se produjo basada en la posibilidad de la obtención de rentas de privilegio. La élite empresaria nacional aprovechó las ventajas excepcionales (subsidios estatales, créditos blandos, promoción industrial, renta del suelo diferencial) para crecer periódicamente en aquellos sectores productivos que fueran beneficiarios de rentas de privilegio en cada momento histórico (producción agroindustrial -tabaco, azúcar, fruticultura, algodón-, cereales en la frontera agropecuaria -sorgo, soja, oleaginosas en general-, armado de aparatos electrónicos, actividad petrolífera, minería, transporte de cargas).

Este modelo interpretativo expresa con claridad lo que ha sucedido en la última década en el ámbito nacional y regional: de una economía en expansión en los primeros años de la Convertibilidad (favorecida por las oportunidades de inversión gestadas por las privatizaciones y la desregulación), se desemboca en otra recesiva que se inicia hacia 1995 con el agotamiento del proceso privatizador y la "Crisis del Tequila", cuya manifestación es el incremento del desempleo, los desequilibrios fiscales en las provincias, el endeudamiento generalizado de los estados provinciales y de las pequeñas y medianas empresas; llegando al estado de recesión de este fin de siglo en que prácticamente no existen, salvo para las actividades privatizadas, opciones productivas rentables y viables. 
Diciembre de 1999 indicará en Argentina el primer cambio democrático de un gobierno justicialista a otro radical en un contexto económico recesivo, marcado por el mayor volumen histórico de déficit fiscal (discutiéndose si es de 4500 o 6500 millones de dólares) y por el grave estancamiento de toda la producción nacional agropecuaria e industrial, afectada por la disminución de la demanda, de los precios y de los volúmenes producidos. Notándose, antes de las elecciones, una disconformidad social más extendida. Si bien ha habido expresiones de protesta a lo largo del último lustro, en el primer semestre fueron más generalizadas, sectorial y regionalmente (La Nación, 17/4/1999 -Sección Campo-; Clarín, 13/6/1999).

En el acápite siguiente nos detendremos en las particularidades de los procesos socioeconómicos que más influencia tienen en la estructuración de la cuestión regional de la actualidad.

\section{Efectos socioeconómicos y territoriales resultantes de la política neoliberal}

El análisis regional del presente y la viabilidad de un crecimiento sostenido y difundido territorialmente, requieren de una mayor comprensión de las transformaciones operadas en la década de los noventa en los ámbitos locales y regionales, resultantes de la globalización y del ajuste macroeconómico neoliberal.

Los procesos que han tenido mayor influencia sobre la conformación territorial de las provincias y regiones del interior argentino son:

1. La privatización de empresas públicas y la desregulación de las actividades productivas, con su consecuente proceso de concentración económica.

2. Las transformaciones en el sector agropecuario provenientes de la entrada de nuevos actores - muchos, inversionistas extranjeros-, crecimiento de otros y desplazamientos de pequeños y medianos.

3. Las grandes inversiones urbanas, extranjeras en su mayoría, que han producido desplazamiento y ruptura del sector minorista e industrial MIPyME (micro, pequeño y mediano), y graves trastornos ambientales y urbanísticos. 
4. El surgimiento y consolidación del Mercosur como área de integración fronteriza, con sus marchas y contramarchas, y con sus beneficios centrados en determinados grupos económicos y zonas fronterizas.

5. Las crisis provinciales, expresión del agotamiento del modelo productivo tradicional y de sus faltas de receptividad y de respuesta al incremento de la desocupación; y no solamente del incremento del déficit fiscal y financiero y de la deuda pública (como suelen sostener desde el Ministerio de Economía de la nación).

No mencionamos entre estos puntos a la notable dinámica adquirida por la minería porque, hasta ahora, sus efectos aparecen muy restringidos en términos territoriales y poblacionales; aunque en torno a la minería se produjo la mayor inversión extranjera en el ámbito nacional: 1200000000 (con capitales australianos, principalmente, y canadienses) en Bajo la Alumbrera (provincia de Catamarca). Ésta es la más importante mina de oro y cobre del país (descubierta hasta el presente) y localizada en el noroeste argentino. Inaugurada en noviembre de 1997 (la etapa de instalación se inició en 1995), se calcula que exportará unos 600000 dólares anuales en mineral en bruto. Depende de las políticas públicas que se implanten, que estos emprendimientos beneficien efectivamente a la población local y al desarrollo de la economía provincial y regional -o que sólo se refleje su actividad por medio del crecimiento del PBI provincial.

La etapa aceleradora para las comunidades locales fue la construcción de la obra civil, que requirió 6000 obreros, cuyos consumos activaron la economía de los pueblos circundantes, como Belén. Pero hoy día los pobladores locales comienzan a observar los escasos -y a veces inexistentes- efectos multiplicadores de esta actividad (un mundo aparte que funciona casi sin vinculación con el exterior). ${ }^{4}$

\footnotetext{
${ }^{4} \mathrm{El}$ mineral no se industrializa en la mina. De La Alumbrera sale un concentrado negruzco mezclado con agua que viaja a Tucumán por un mineraloducto de $314 \mathrm{~km}$; allí se extrae el agua y se obtiene una arena oscura que se transporta por tren, en vagones propios de La Alumbrera, hasta el puerto privado de San Martín, al norte de Rosario. Y de allí se embarca al extranjero, porque la extracción final del cobre y del oro se hace en países como los Estados Unidos, Japón, Brasil, Canadá, Corea, España (Clarín, 23/11/ 1997). Como puede verse, en este recorrido todos los nodos de intercambio pertenecen a la propia empresa (no hay articulaciones con otras actividades o efectos multiplicadores).
} 


\section{Las privatizaciones y las desregulaciones}

Privatizaciones y desregulaciones fueron el antecedente de un proceso de concentración capitalista en abundancia y de extranjerización de la economía nacional. ${ }^{5}$ El capital internacional se asoció con importantes grupos representativos de la cúpula empresaria nacional, en buena medida para aprovechar sus relaciones y vínculos con el poder político.

La magnitud de la concentración capitalista operada consolidó, como nunca antes, el control de los principales resortes del poder político y económico por parte del capital más concentrado. Uno de los canales por los que se encauzó este proceso fue la garantía implícita de una muy alta tasa de rentabilidad sobre el capital invertido y sobre las ventas. En el caso de las privatizaciones esta rentabilidad resultó muy superior a la media nacional, constituyéndose, por ello, en el sector de inversión más rentable del país (Aspiazu, 1997:14 y ss.). Y ello fue posible porque las empresas privatizadas o concesionadas operaron con un mercado cautivo, y recibieron garantías, atribuciones y ventajas en precios y condiciones -muchas en áreas estratégicas de recursos naturales, transporte, energía, comunicaciones-. Es más: “[...] se trata de empresas que han logrado privilegios que forman parte de una especie de barrera no sólo al ingreso, sino también a las crisis" (Aspiazu, 1997:31). Afirmación que resulta de observar que durante “el Tequila”, en 1995, pudieron mantener, y aun acrecentar sus ganancias.

Las privatizaciones en el interior significaron la pérdida de las únicas o principales fuentes de empleo existentes en numerosas localidades pequeñas y medianas; una consecuencia fue la transformación territorial de las localidades en que se asentaban dichas actividades, así como también de los ámbitos aledaños conectados a las mismas. Algunas se transformaron en pueblos fantasma, otras perdieron su dinámica económica, proliferando las actividades informales y los pequeños comercios precarios e, incluso, ilegales.

Quizás el caso más paradigmático sea la privatización de Yacimientos Petrolíferos Fiscales (YPF) operada en 1991-1992, que afectó a numerosas localidades vinculadas a esta actividad. Como

${ }^{5}$ APyME (1999:152), a partir del informe anual de 1998 de la CEPAL, precisa la considerable multiplicación de las empresas extranjeras entre las 500 más grandes de Argentina: en 1990 había 116 empresas extranjeras que generaban 34\% de las ventas; en 1995 ya había 214 con 51\% de la facturación, y en 1997, 244 con 57\% de ésta. 
sucedió en Salta con Tartagal (43 570 habitantes en 1991), General Mosconi (11 108 habs.) y Campamento Vespucio (1 535 habs.); en Neuquén, con Cutral-Có y Plaza Huincul (44 806 habs.); y con todas las de la cuenca del Golfo San Jorge: Comodoro Rivadavia (124 121 habs.) en Chubut; y en Santa Cruz, Caleta Olivia (27 946 habs.), Pico Truncado (7 091 habs.) y Las Heras (6 333 habs.). Otros ejemplos son la privatización de Yacimientos Carboníferos Fiscales (YCF) en 1992, actividad central en la cuenca de Río Turbio en Santa Cruz, con eje en la localidad Yacimientos Río Turbio (6 753 habs.) (Salvia y Panaia, 1997).

Los efectos de estos procesos privatizadores sobre los mercados de trabajo y sobre la conformación urbana de las localidades involucradas, también se repiten cuando se produce el cese, cierre, venta de actividades que eran las mayores demandantes de empleo en sus respectivas zonas (y que desaparecen o se reestructuran como resultado de la modernización, de la competitividad y de la renovación tecnológica exigida por la globalización). Esto sucedió con la producción de acero en: Sierra Grande (11 213 habs.), Río Negro; con los Altos Hornos Zapla en Palpalá (39000 habs.), Jujuy; con la licitación y venta a un grupo extranjero del Ingenio San Martín de Tabacal en Salta, vinculado a las localidades de Pichanal (10 903 habs.), e Hipólito Yrigoyen (8 539 habs.) y El Tabacal (1 113 habs.); o con la reestructuración del Ingenio Ledesma en Jujuy.

De aquí se explican las razones en estas localidades, y en muchas otras, de las manifestaciones populares o los estallidos sociales de protesta (véase Carrera y Cotarelo, 1997 y 1998). La población, por medio de cortes de ruta, revueltas populares, expresa su descontento y oposición a la política económica de ajuste y a sus consecuencias sociales y políticas. Se opone a los despidos masivos, a las reducciones de sueldos, a la caída del consumo; a la quiebra de pequeñas y medianas empresas y comercios ligados a las actividades centrales; al cobro de mayores tasas por parte de las empresas de servicios privatizadas (electricidad, gas, agua, teléfonos); a los gobiernos provinciales clientelísticos y corruptos, asociados con los intereses de los grupos hegemónicos locales (las privatizaciones son una fuente privilegiada de generación de recursos ilegales para tergiversar las decisiones a partir del soborno).

Por su parte, la desregulación (otra medida resultante del ajuste) impulsa al incremento de la concentración económica, porque el "libre juego" de la oferta y la demanda implica que las 
reglas de funcionamiento del mercado quedan "liberalizadas", es decir, libradas al accionar de quienes tienen el poder económico, financiero y productivo sobre el mercado. Más grave aun cuando esto sucede inmerso en el proceso de globalización y de transnacionalización, ya que el mercado está dominado por grupos económicos que manejan estrategias empresariales multiproducto y multirregiones, y dificultan el acceso de aquellos países cuya base productiva se sustenta en la explotación agropecuaria individual (León, 1994:89).

La desregulación comenzó con la desaparición de la Junta Nacional de Granos que garantizaba precio mínimo y sostén a la producción de cereales y oleaginosas, beneficiando en especial a los productores pequeños y medianos de la pampa húmeda. Pero luego continuó con la disolución o el desmembramiento de los entes reguladores de las producciones regionales de yerba mate, vino, azúcar y tabaco. Estos entes buscaban proteger al productor, especialmente al pequeño, frente a la industria y su influencia en la determinación del precio al productor. Hoy día todas estas producciones se encuentran afectadas por la caída de precios y por el avance de grandes capitales o grupos económicos agroindustriales, nacionales e internacionales, en el control de los respectivos mercados, con la consiguiente desprotección de los pequeños y medianos agropecuarios.

Además, tanto la apertura comercial como la integración con Brasil obligan a la reconversión de muchos cultivos regionales. En este contexto, el cese del sistema regulatorio acelera la reconversión productiva, pero no para todos los productores, porque no todos están en condiciones de llevarla a cabo, como sucede con los pequeños productores (y muchos de los medianos) en un contexto de creciente concentración económica. Volveremos sobre este proceso de concentración al tratar el sector agropecuario y las inversiones en el ámbito urbano.

Otras repercusiones territoriales desfavorables resultantes de las privatizaciones pueden sumarse a los factores enumerados. Los peajes en rutas, nacionales y provinciales, contribuyeron a incrementar los costos de producción y traslado en general, pero aún más para los productos y pasajeros de las economías regionales distantes del principal mercado de consumo interno -el Gran Buenos Aires-, que incluso funciona como puerto del mercado internacional. Los diferentes ramales de la red privatizada convergen en forma radial hacia este mercado y su puerto. Los 10 mil km de rutas nacionales privatizadas y los accesos a la Capital 
Federal concentran la mayor parte de la producción, de los insumos y de las personas que se movilizan en el territorio argentino.

El pago del peaje implica un costo agregado de traslado y aunque se justifica con la reducción de otros gastos para los usuarios (como ahorro en tiempo de viaje, cubiertas, combustible y desgaste del vehículo), el mismo no ha sido evaluado. ${ }^{6}$ El peaje se incrementó en términos reales, cualquiera que sea el índice de referencia que se coteje. En diciembre de 1997 la tarifa promedio era de $\$ 1,70$ por cada $100 \mathrm{~km}, 55 \%$ más que la tarifa media del primer periodo tarifario iniciado desde la Convertibilidad (abril 1991-julio 1992); y en este último los incrementos reales fueron: 17\% con relación al Índice de Precios al Consumidor, 12\% al Índice de la Construcción, y 32\% al Índice de Precios internos al por mayor (Arza y González, 1998:48).

Pero además, con el surgimiento del plan de convertibilidad (y a menos de cuatro meses de la concesión) se produjo la primera renegociación. ${ }^{7}$ Ésta implicó la suspensión del pago del canon por parte de las empresas, que también recibieron compensaciones indemnizatorias, otorgadas por medio del presupuesto de la Dirección Nacional de Vialidad (Arza y González, 1998: 59). ${ }^{8}$ Estas modificaciones tienen una consecuencia importante para el análisis territorial. La anulación del canon y el pago de las indemnizaciones implicó que no se invirtiera en mejoras y ampliación de las rutas no concesionadas (porque dichos recursos fueron destinados a las empresas concesionarias). Las privatizaciones llevaron a la caída de la inversión pública en carreteras en general, al desmantelarse el sistema previo de financiamiento del sector por medio de los impuestos a los combustibles.

$\mathrm{Al}$ respecto, nótese que las rutas nacionales tienen una extensión de 38700 km (80\% pavimentados -Clarín, 17/5/1998-) y las concesionadas sólo son $1 / 3$ de las nacionales pavimentadas. Precisamente, en la mayor parte de los $2 / 3$ restantes las condiciones de transitabilidad son deficientes, dada la ausencia de inver-

${ }^{6}$ No existen análisis oficiales que estimen el ahorro efectivo que obtiene el usuario. Otros estudios disponibles indicarían que en la mayor parte de los corredores viales, el ahorro neto del usuario es nulo o negativo (Arza y González, 1998:55)

${ }^{7}$ La discontinuidad regulatoria es una característica del sector (tanto en relación con las tarifas como con las inversiones comprometidas y con los plazos de concesión).

${ }^{8}$ Es importante resaltar que la totalidad de las firmas que forman parte de los consorcios adjudicatarios, son las antiguas empresas contratistas del Estado, mediante la Dirección Nacional de Vialidad. Es decir, se trata de empresas con una antigua relación con el Estado y con mucha experiencia en el ejercicio del lobby empresarial. Quizás aquí pueda encontrarse algún elemento que sirva para entender las condiciones favorables y las ventajas obtenidas por las empresas en sus negociaciones con el Estado. 
siones y mejoras. ${ }^{9}$ Este estado tiende a deteriorarse aún más a medida que subsiste el ajuste y se profundiza la recesión.

\section{Las transformaciones del sector agropecuario}

En el sector agropecuario la apertura comercial implicó: (i) la eliminación de las retenciones a la exportación; (ii) la desgravación a la importación de bienes de capital y de insumos; (iii) menores aranceles para importar productos competitivos.

Estas medidas, en general, estuvieron destinadas a mejorar los precios al productor (como porcentaje de los precios internacionales) y a disminuir los costos de producción. Pero esto, sin embargo, beneficia sobre todo a los productores de mayor tamaño; no así a los pequeños y medianos a quienes no sólo no beneficia sino que frecuentemente perjudica, por la competencia de productos importados y por las menores posibilidades de conseguir fuentes alternativas de ingresos, vía el empleo estacional en el sector agropecuario.

La desgravación de los bienes de capital fue aprovechada por quienes están en condiciones de desarrollar un proceso amplio de mecanización, beneficiándose con los efectos resultantes: mejoras de calidad, incremento de la productividad y sustitución de mano de obra por capital. Por su parte, la disminución de aranceles a la importación de productos competitivos, no condiciona el desarrollo de quienes tienen posibilidad de reconversión productiva, y pueden adecuarse en forma continuada y permanente a las condiciones cambiantes de demanda y calidad del mercado internacional. Pero ésta tampoco es la situación general de los pequeños y medianos productores.

Un análisis particularizado por producto ${ }^{10}$ indicaría que el aumento de las exportaciones regionales operado, no siempre resultó en una mejora en la balanza comercial respectiva (exportaciones menos importaciones), como en el caso de las frutas y hortalizas elaboradas, en el que ha habido una elevada importación. En estos casos es el sector pequeño-productor, que comercializa en el mercado interno, el que resulta afectado por la mis-

\footnotetext{
${ }^{9}$ Esta situación se repite en las rutas provinciales, inclusive con mayor gravedad, dado el deterioro más agudo de las economías provinciales (la extensión de las rutas provinciales es de $176330 \mathrm{~km}, 21 \%$ pavimentado, 24\% mejorado y $55 \%$ de terracería).

${ }^{10}$ Un análisis respecto al producto tiene sus dificultades, dado el déficit existente en Argentina en las estadísticas de producción, importación y exportación desagregadas por rubros comparables (León, 1994:83).
} 
ma, debido a las dificultades de competir en calidad y precios, y por la consecuente caída en los precios de sus productos.

Socialmente esto significa, a mediano plazo, el desplazamiento de muchos de ellos, con la consecuente mudanza del trabajo por cuenta propia a la desocupación o a una forma de trabajo informal más precario. Incluso peor, dada la intensa mecanización en ciertas producciones (como el azúcar y el algodón) que lleva a la extinción del empleo rural.

No obstante, para la economía en su conjunto estas medidas llevaron: (i) a un notorio incremento de la producción agrícola pampeana de granos y oleaginosas (también en varias provincias del noroeste y noreste que continuaron con el proceso de "pampeanización" ${ }^{11}$ ); y (ii) a una expansión de muchos cultivos regionales, varios vinculados al mercado externo. Así sucedió en las provincias del noreste con el algodón, el té y el arroz; y en las del noroeste con las hortalizas, las legumbres, el algodón, las frutas frescas, los cítricos y el tabaco. Mereciendo una especial mención el limón de Tucumán, el algodón de Santiago del Estero y Chaco, y el arroz de Corrientes y Entre Ríos, por sus importantes incrementos productivos, su destino para la exportación -fuerte anclaje en el Mercosur-y sus efectos sobre las economías provinciales respectivas. ${ }^{12}$

Así mismo, cabe señalar que estos incrementos productivos también estuvieron vinculados con un continuado avance de las grandes inversiones en tierras, favorecidas por los bajos precios de las mismas. Estas grandes inversiones se han expandido considerablemente por todo el territorio nacional, llegando dos fir-

${ }^{11}$ Se denomina así "a la expansión de la frontera agrícola hacia el chaco salteño. Las provincias de Salta, Santiago del Estero y Formosa comenzaron, al promediar la década de los setenta, a producir (por corrimiento del ciclo húmedo) productos típicamente pampeanos, como cereales y oleaginosas (maíz, soja, sorgo, trigo).

${ }^{12}$ En la campaña 1996/97 la cosecha de arroz superó 1200000 toneladas con una superficie de 226000 hectáreas (ha), mientras que la media del trienio 1990/91-1992/ 93 fue de 557000 toneladas y 129100 ha (Carballo y Pagliettini, 1998:3). Esto representa un incremento promedio de $115 \%$ y $75 \%$, respectivamente. En algodón, Argentina está a la cabeza de la expansión mundial. En 1995/96 se llegó al millón de hectáreas sembradas (habiéndose expandido más de 200\% respecto a 1992/93, ya que 316000 ha fue la superficie sembrada cuatro campañas antes). Este crecimiento se dio a partir de grandes explotaciones algodoneras (aparecieron productores con 1000 y 5000 ha totalmente mecanizadas -y hasta algunos megaproductores con 10000 y 20000 hacuando en etapas anteriores la superficie media de este cultivo no superaba las 20 ha -Valeiro, 1997-). Por su parte, la producción de limones de Tucumán es de alta tecnología, con predominio de firmas grandes, con una firme y creciente inserción en el mercado internacional y que emplea significativa cantidad de mano de obra (a pesar de la mecanización). La producción de limón en Tucumán pasó de 468560 toneladas en 1992/93 a 720000 toneladas en 1996/97 (lo que representa 80\% de la producción nacional). 
mas extranjeras (Cresud Agropecuaria -representante del grupo Soros-y Benetton) a constituirse en el ámbito nacional en la mayor propietaria de tierras y de cabezas de ganado, respectivamente. ${ }^{13}$

Conjuntamente, la política de ajuste ha llevado a la quiebra a muchos pequeños y medianos productores, porque además de lo ya mencionado arriba, el ajuste careció de una política crediticia para este sector obligado a la reconversión. El crédito sólo existió para quienes tenían garantías reales y acceso facilitado al poder político y al sector financiero. Un ejemplo de ello son los "diferimientos" (crédito subsidiado) cuyos beneficiarios son exclusivamente los grandes inversionistas, porque los pequeños y medianos no alcanzan las condiciones mínimas de solvencia y capacidad contributiva acordes con el monto del crédito. ${ }^{14}$

\section{Las transformaciones urbanas}

Las grandes inversiones urbanas con frecuencia desplazaron al gran capital local -o a veces se asociaron con él-. Su proceso de localización comenzó en Capital Federal y el Gran Buenos Aires, para luego expandirse regionalmente hacia las principales ciudades del interior, la mayoría capitales de provincia.

Todas estas grandes inversiones urbanas en general, y los hipermercados en particular, generan un nuevo espacio urbano y periurbano, siendo sus consecuencias inmediatas y más visibles: la pérdida de ocupación en los pequeños establecimientos (empleados, patrones y por cuenta propia); el quiebre comercial de éstos; la segregación residencial y comercial y las transformacio-

${ }^{13}$ Una representante de este grupo inversionista (Clarisa Lifsic-directora de Cresud Agropecuaria-) señaló que: "En Argentina, la otra ventaja que tenemos además de las condiciones de la tierra y el clima, es que el precio de la tierra comparado con otros países del Primer Mundo es mucho menor... a igualdad de rendimientos".

${ }^{14}$ Por ley nacional 22021 se permite a los inversionistas diferir el pago de impuesto al valor agregado y a las ganancias generado por su actividad, para aportarlos a un proyecto que ellos promuevan. El monto que se puede invertir por diferimiento asciende a $75 \%$ del proyecto, y $25 \%$ debe hacerse con inversión propia. Para acogerse al sistema hay que conformar una sociedad anónima y presentar un proyecto (que defina esquema productivo, capacidad económico-financiera de la empresa y rentabilidad factible). Por último, se requiere de la aprobación de la provincia respectiva. Para la empresa promovida la ley otorga un periodo de ejecución del proyecto (5 años), un periodo de gracia ( 5 años), y generalmente en el año 11 se comienza a devolver el impuesto diferido, sin interés, en cinco cuotas anuales (La Nación, 20/6 y 27/6/1998). Se trata en realidad de un crédito a largo plazo con interés cero, del que no pueden beneficiarse los empresarios pequeños y medianos, entre otras razones, porque sus montos de impuestos no son significativos como para permitirles constituir, con éstos, una cantidad de capital equivalente a $75 \%$ de un proyecto de inversión. 
nes urbanísticas sin planificación previa, con sus consecuentes riesgos ambientales, económicos y sociales (dificultades de transporte, aumento de la contaminación ambiental, de los accidentes, incremento de la delincuencia, disminución y destrucción de los espacios verdes y de esparcimiento público masivo, etc.).

Estos procesos territoriales desembocan en urbanizaciones duales, con su respectivo aumento de la polarización social (Borja y Castells, 1998:59 y ss.). Procesos de exclusión, con espacios del mismo sistema urbano desarticulados, con funciones valorizadas y degradadas, con grupos sociales productores de información y tenedores de riqueza, frente a otros marginales y excluidos, son realidades en muchas capitales de provincia y en otras importantes ciudades (como Córdoba, Mendoza, Salta, Rosario, Mar del Plata, Tucumán). En todas ellas se repiten formas de duplicación urbana, contrastes cada vez mayores entre barrios ricos y pobres, polarización entre un sector minoritario de la población con acceso a consumos y servicios valorizados y modernos, frente a otro mayoritario con condiciones de vida en creciente deterioro.

También, y como ya señalamos, el proceso de privatizaciones y la reestructuración productiva resultante del ajuste modificaron la conformación territorial de las localidades del interior, fuertemente articuladas con las actividades privatizadas, modernizadas o transformadas. Es el caso de buena parte de las ciudades nacidas en torno a la explotación de los recursos petrolíferos. Algunas se han convertido casi en pueblos fantasma, como Pico Truncado en Santa Cruz; en otras resalta la proliferación del comercio informal (e incluso ilegal) de diversa índole, como Caleta Olivia en Santa Cruz, y Tartagal, Mosconi y Vespucio en Salta.

Otras transformaciones urbanas se operan en aquellas localidades ligadas a alguna actividad central que, como resultado de la globalización, entra en crisis o ha sido tecnificada, y esto lleva al despido masivo de trabajadores, a la no contratación de personal eventual y a la rebaja del sueldo de los que están empleados. Esta situación modifica el índice de consumo de la población y afecta la dinámica económica de las localidades en que ellos viven, deteriorándose las actividades formales (comercio y servicios) y profundizándose la informalidad, las actividades ilegales, sin control y precarias en sus formas (vendedores ambulantes, ferias, transporte alternativo). Es el caso de las localidades que se desarrollaron, por ejemplo, en torno al Ingenio San Martín de Tabacal (Pichanal, Hipólito Yrigoyen, El Tabacal). 


\section{El Mercosur}

El surgimiento del Mercosur constituye el modelo de integración subregional de la década de los noventa. El mandato para entrar y sostenerse en éste es la búsqueda de la "competitividad", y se ha constituido en el paradigma, en el que productores y empresas deberían participar para no excluirse del proceso de desarrollo y crecimiento económico.

Sin embargo, los beneficios resultantes del Mercosur (el incremento de los intercambios comerciales y de los flujos en general) han constituido, por ahora, un gran negocio para las grandes empresas, atraídas por la magnitud del mercado de demanda potencial que se vislumbra. El caso más claro es el de la industria automotriz, una verdadera creación de "industria del Mercosur", que sólo se justifica por un mercado de 200000000 de personas con una capacidad adquisitiva que puede superar los 3000000 de automóviles al año 2000, comparable al mercado de Alemania, el mayor de Europa y uno de los mayores del mundo (Sartelli, 1997:52).

Para los productores de las distintas provincias, la participación en estas áreas de integración fronteriza depende no sólo de la disponibilidad de capital y tecnología, también de la localización espacial, que implica superar distintas barreras al acceso al transporte en general (sobre todo vial, ferroviario y fluvial). De hecho se está dando una revalorización estratégica del espacio geográfico limítrofe con el Mercosur que conduce a notorios cambios territoriales, como es el caso de las provincias del noreste en donde se vienen ejecutando y proyectando numerosas obras de integración vial y fluviales sobre los ríos Paraguay y Paraná. ${ }^{15}$

Así mismo, el comercio externo con el Mercosur ha crecido notablemente para muchas provincias, siendo un signo de la senda futura de las exportaciones provinciales. Entre 1993 y 1996 las exportaciones nacionales al Mercosur crecieron más del doble (pasaron de casi 3700 millones a más de 7900 millones) y 18 de las 24 jurisdicciones incrementaron su participación en el Mercosur (excepto Formosa, Jujuy, Neuquén, Tucumán, San Juan y Tierra del Fuego).

De todos modos, debe tenerse en cuenta que el Mercosur, si bien es un proceso en marcha, suele estar lleno de incertidum-

\footnotetext{
${ }^{15}$ Corrientes y Misiones limitan con tres estados de Brasil -Santa Catarina, Paraná y Rio Grande do Sul- cuya población es igual a la de toda Argentina.
} 
bres, pues depende del grado en que se respeten los acuerdos, y ante todo, del mantenimiento de la estabilidad económica en los países miembros (es decir, el control de índices de inflación mínimos en ambos países, que no tienen economías consolidadas como las de la Unión Europea). Mercosur significa para Argentina: mantenimiento de la performance económica con Brasil, con toda la imponderabilidad política y económica de la misma.

\section{Ajuste fiscal y crisis provinciales}

Las administraciones de la mayoría de las provincias argentinas han funcionado, antes y después de la restauración democrática, con estrechos vínculos con el poder económico provincial tradicional (representantes de los grupos que controlan las actividades tradicionales como la producción de azúcar, tabaco, algodón, vid, yerba mate, té, la fruticultura de los valles subtropicales, etc.). Estos sectores, por su propia lógica de funcionamiento, han antepuesto sus intereses a los de la región donde se localizan sus actividades.

Es frecuente que los beneficios que obtienen por ciertas emergencias o desventajas regionales, los utilicen con otros fines distintos a la inversión de los mismos regionalmente, ya sea para reconvertir o para avanzar en la competitividad de la producción local. Ejemplos de ello abundan, como sucede con los redescuentos que se otorgan, por zona de emergencia, y se utilizan para valorizarlos financieramente en la Capital Federal; o con la condonación de deudas a todos los productores -grandes y pequeños-, operada durante la dictadura militar (gestión de Domingo Cavallo al frente de Banco Central), cuyos beneficios se reinvirtieron en el extranjero (así ocurrió con una de las mayores empresas de la fruticultura del valle del Río Negro, que en esos años instaló una planta productora en Brasil).

Es común que las gobernaciones provinciales transfieran recursos, tributarios o financieros, a los sectores económicos dominantes en el ámbito provincial, y éstos no los utilicen en el desarrollo productivo local alternativo, en la reconversión, sino en la obtención de rentas de privilegio y de corto plazo. Entre las reformas financieras de 1977 y 1985, las gobernaciones provinciales asistieron sistemáticamente a los sectores tradicionales de sus regiones, con una política que no buscaba planificar el desarrollo, pues ignoraba los cambios en las condiciones productivas 
y financieras (costo y plazo del financiamiento). El resultado fueron carteras irrecuperables que al final se licuaron con la estatización de las deudas empresariales operada desde 1981 (IDEP-ATECTA (1996:3). ${ }^{16}$

En 1991 se produce una primera fase del ajuste provincial con la negativa del Banco Central a continuar asistiendo a la banca oficial de las provincias por su estado de insolvencia y falta de operatividad (lo cual terminó con la liquidación del Banco de La Rioja). La segunda fase fue la transferencia a las provincias de los gastos sociales (educación y salud), una consecuencia del ajuste nacional. Para flexibilizar el gasto nacional se eliminaron o privatizaron sus componentes rígidos (empresas públicas, organismos de regulación), y en los casos en que esto no fue posible se descentralizó hacia las provincias. Es lo que sucedió con la transferencia de la infraestructura social (escuelas, hospitales) y la parte de las empresas públicas no privatizables (ramales ferroviarios no rentables, obras de riego). ${ }^{17}$ En la tercera fase, primero se recortó la participación en los recursos coparticipados (Pacto Federal Ley 24.130/1992), y luego se limitó el poder tributario provincial (Pacto Federal para el Empleo, la Producción y el Crecimiento -decreto 1807/1993-).

Los recortes sufridos por las provincias no se percibieron inmediata y directamente en esos años, ya que el monto total a distribuir aumentó como resultado de la mayor dinámica del mercado interno del principio de los noventa y por la mejor captación de impuestos. Es decir, estas transformaciones en la relación nación-provincias se ejecutaron entre 1991 y 1994, en un contexto de abundancia de recursos fiscales. La expansión del gasto público nacional (favorecido por las privatizaciones) y más aún del gasto presupuestario provincial (resultado del incremento de la recaudación impositiva, asociado a la expansión del consumo interno durante la primera etapa del gobierno de Menem), fue lo que persuadió a las provincias a aceptar las duras condiciones impuestas.

Durante esos mismos años las provincias aumentaron el empleo público y, de este modo, afrontaron la caída en la demanda de fuerza de trabajo por parte de los sectores productivos. El empleo público se expandió de modo considerable (casi 700000

\footnotetext{
${ }^{16}$ Parte de los párrafos que siguen se basan en IDEP-ATE-CTA, 1996, p. 3 y ss.

${ }^{17}$ El Estado nacional privatizó las partes rentables de los ferrocarriles y de Agua y Energía, y delegó en las provincias la voluntad de mantener o no lo que no fuere rentable bajo su propio riesgo y administración.
} 
empleados provinciales en 1983 frente a más de 1000000 en 1995).

Por todo esto, los conflictos provinciales que se sucedieron en el lapso 1991-1994, se vincularon esencialmente con la falta de horizonte productivo en general y con la corrupción generalizada en la mayoría de las administraciones provinciales (como en el caso de Santiago del Estero). En cambio, las crisis provinciales del presente tienen su origen en la fase recesiva en que ha entrado la convertibilidad, luego de la caída en el ingreso de capitales por el agotamiento del proceso privatizador. Lo cual se traduce en menores oportunidades laborales, recesión en el mercado interno, quiebra de pequeños y medianos productores, de comerciantes y empresarios, e inviabilidad para actividades económicas opcionales.

En este contexto recesivo y con el anclaje de la convertibilidad, el endeudamiento creciente es el recurso encontrado por las administraciones provinciales para encargarse del equilibrio de las cuentas fiscales, de las prestaciones sociales (salud, educación, cultura), de subsidiar a los servicios públicos no rentables (transporte, energía, riego, comunicaciones), y para afrontar el desempleo con la carga del empleo público. Y al no tener acceso al Banco Central, acuden a la banca privada y a los organismos internacionales -cuyas condiciones son más dificiles y restrictivas. Ello implica un endeudamiento más costoso y con garantías reales, que surgen de comprometer su principal y más seguro recurso: la coparticipación. ${ }^{18}$

\section{Una reflexión final}

En la medida que el ajuste fiscal y económico no se aplica de modo diferencial para posibilitar en las provincias más desfavorecidas un desarrollo productivo alternativo, nos preguntamos ¿no será que, de manera subyacente, a determinadas unidades provinciales se las está considerando inviables para adecuarse a las demandas del actual contexto de globalización?

No se discute que la reconversión de las economías regionales resulte hoy día un requisito impostergable para la inserción

${ }^{18}$ Para los bancos prestatarios la coparticipación constituye una garantía real, en tanto tienen asegurado su cobro mediante la afectación que de la misma hace el Banco Central. Es decir, el monto adeudado no se les gira a las provincias, pues pasa directamente del Banco Central a los acreedores bancarios. 
económica y productiva de las provincias en el contexto de desarrollo actual. Empero, dada la situación socioeconómica de las provincias y del país, así como el modelo de política económica y las reglas de juego imperantes, se vislumbra que la "reconversión", en realidad, tiene un solo destinatario: los grandes grupos capitalistas nacionales que necesitan reconvertir para adecuarse a las demandas del mercado internacional. Por lo tanto su destino será, de nuevo, aumentar y exacerbar el proceso de concentración económica. En otras palabras, la "reconversión" y el consecuente objetivo de alcanzar la "competitividad sistémica" (como demanda excluyente de la globalización en las provincias), irán de la mano de los grandes capitales, no de los pequeños ni de los medianos.

Más aún, cuando se observa que hasta la actualidad, más que avanzar hacia "la competitividad sistémica", se produce una generalizada pérdida de la misma en la mayoría de las actividades regionales a causa de: (i) la fijación del peso al dólar frente a las fluctuaciones -evaluaciones y revaluaciones- de las monedas de otros países con los que mantenemos vínculos comerciales (dólar, yen, marcos alemanes, libras esterlinas, real); (ii) las altas tasas de interés en términos reales; (iii) el incremento de tarifas y peajes posterior a las privatizaciones de los servicios públicos; (iv) la deflación de precios del principal socio del Mercosur (luego de la crisis financiera de Brasil producida el segundo trimestre de 1999); y (v) la caída de la mayoría de los precios de los productos regionales. Condicionamientos que llevan a la merma de la rentabilidad de las actividades comerciales en general, y restringen el crecimiento productivo de las provincias. El impacto resulta inmediato: el aumento de la desocupación (con su consecuente polarización social).

Por otra parte, el crecimiento de la primera mitad de los años noventa no trajo aparejado desarrollo, redistribución. El aumento de la desocupación en los aglomerados provinciales lo prueba. El desempleo pasó de un dígito histórico a dos dígitos a mediados de los noventa y desde entonces se mantuvo estable (salvo escasas excepciones como Formosa). Esto es el resultado del impacto social desfavorable de las reestructuraciones de las empresas públicas y privadas que llevó a una creciente tecnificación con desocupación. Sumándose además el incesante deterioro de la situación fiscal y de endeudamiento de los estados provinciales, que los sucesivos ajustes nacionales y provinciales no solucionaron (dada la recesión que implican). 
Concluyendo, el contexto de ajuste y convertibilidad no hace más que acentuar las desigualdades socioeconómicas regionales, pues agranda la brecha entre las provincias ricas y las pobres. Las más desfavorecidas, en recursos naturales y económicos, con un perfil productivo acotado, no pueden (i) afrontar la rigidez de sus gastos públicos y las restricciones que les impone la convertibilidad, ni (ii) mantener la ocupación pública como fuente de captación de los desocupados. Por otra parte, (iii) sus producciones tradicionales desestructuradas tampoco tienen capacidad para captar a los desocupados, y cada vez más, (iv) las nuevas actividades que aparecen, o las que se reestructuran, lo hacen a partir de un modelo productivo caracterizado por mínima ocupación de mano de obra.

En otras palabras, este contexto socio-político y económico pone en duda que las provincias más pobres cuenten con capacidad empresarial y estructura socio-productiva y física para la reconversión y el desarrollo económico. 
Cuadro 1

Argentina, por jurisdicción (ordenadas de mayor a menor según el $\mathrm{PBG} /$ habitante).

Producto Geográfico Bruto por habitante en 1996.

Población con Necesidades Básicas Insatisfechas (NBI) en 1991 (en \% sobre la población total)

\begin{tabular}{|l|r|r|}
\hline \multicolumn{1}{|c|}{ Jurisdicción } & $\begin{array}{c}\text { PBG/hab. año 1996, } \\
\text { en \$ }\end{array}$ & NBI 1991 \\
\hline Ciudad de Buenos Aires & 21970 & 8.1 \\
\hline Santa Cruz & 14371 & 14.7 \\
\hline San Luis & 13102 & 21.5 \\
\hline Tierra del Fuego & 12015 & 22.4 \\
\hline Chubut & 10199 & 21.9 \\
\hline Neuquén & 8519 & 21.4 \\
\hline La Pampa & 8246 & 13.5 \\
\hline La Rioja & 7854 & 27.0 \\
\hline Santa Fe & 7146 & 17.6 \\
\hline Buenos Aires & 7090 & 17.2 \\
\hline Río Negro & 6906 & 23.2 \\
\hline Córdoba & 6827 & 15.1 \\
\hline San Juan & 4910 & 19.8 \\
\hline Entre Ríos & 4870 & 20.6 \\
\hline Misiones & 4689 & 33.6 \\
\hline Mendoza & 4458 & 17.6 \\
\hline Catamarca & 3824 & 28.2 \\
\hline Salta & 3771 & 37.1 \\
\hline Tucumán & 3561 & 27.7 \\
\hline Corrientes & 3233 & 31.4 \\
\hline Chaco & 2930 & 39.5 \\
\hline Jujuy & 2802 & 35.5 \\
\hline Formosa & 1996 & 39.1 \\
\hline Santiago del Estero & 1978 & 38.2 \\
\hline Fur Earara & $386 r$ & \\
\hline
\end{tabular}

Fuente: Elaboración propia con datos de Informe sobre las Provincias Argentinas, Buenos Aires, Ministerio del Interior, 1999. 
Cuadro 2

Argentina, Índice de Desarrollo Humano (IDH) por jurisdicciones en 1995 (estimado)

\begin{tabular}{|c|c|c|}
\hline Jurisdicción & IDH 1995 & Núm. de orden \\
\hline Ciudad de Buenos Aires & 0.923 & $1^{\circ}$ \\
\hline Córdoba & 0.896 & $2^{\circ}$ \\
\hline Tierra del Fuego & 0.895 & $3^{\circ}$ \\
\hline Santa $\mathrm{Fe}$ & 0.894 & $4^{\circ}$ \\
\hline Santa Cruz & 0.891 & $5^{\circ}$ \\
\hline Buenos Aires & 0.888 & $6^{\circ}$ \\
\hline Mendoza & 0.886 & $7^{\circ}$ \\
\hline La Pampa & 0.883 & $8^{\circ}$ \\
\hline La Rioja & 0.879 & $9^{\circ}$ \\
\hline Neuquén & 0.878 & $10^{\circ}$ \\
\hline Chubut & 0.876 & $11^{\circ}$ \\
\hline Entre Ríos & 0.876 & $12^{\circ}$ \\
\hline San Luis & 0.875 & $13^{\circ}$ \\
\hline Río Negro & 0.873 & $14^{\circ}$ \\
\hline San Juan & 0.873 & $15^{\circ}$ \\
\hline Catamarca & 0.850 & $16^{\circ}$ \\
\hline Misiones & 0.840 & $17^{\circ}$ \\
\hline Tucumán & 0.828 & $18^{\circ}$ \\
\hline Salta & 0.813 & $19^{\circ}$ \\
\hline Corrientes & 0.791 & $20^{\circ}$ \\
\hline Jujuy & 0.763 & $21^{\circ}$ \\
\hline Chaco & 0.758 & $22^{\circ}$ \\
\hline Santiago del Estero & 0.735 & $23^{\circ}$ \\
\hline Formosa & 0.732 & $24^{\circ}$ \\
\hline Total país & 0.887 & \\
\hline
\end{tabular}

Fuente: Honorable Senado de la Nación, Informe Argentino de Desarrollo Humano, Buenos Aires, 1998 (con datos del INDEC y del Ministerio del Interior). 


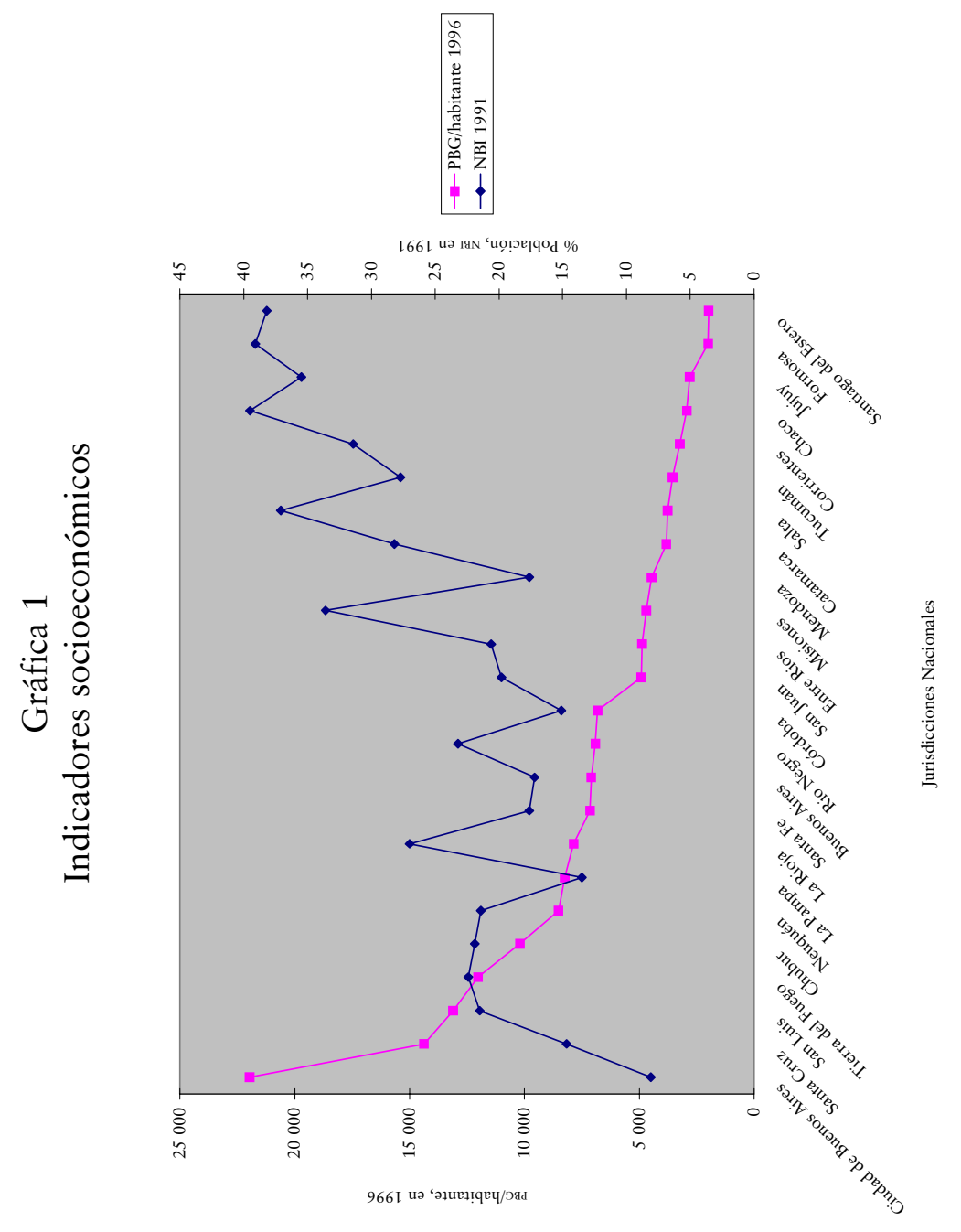




\section{Bibliografía}

Аруме (1999), “Extranjerización de la economía argentina”, Realidad Económica, núm. 163, pp. 152-159, Buenos Aires, IADE.

Arza, Camila y Angelita González García (1998), "La privatización del sistema vial: historia de un fracaso", Realidad Económica, núm. 156, pp. 38-61, Buenos Aires, IADE.

Aspiazu, Daniel (1997), "El nuevo perfil de la elite empresaria. Concentración del poder económico y beneficios extraordinarios", Realidad Económica, núm. 145, pp. 7-32, Buenos Aires.

Borja, J. y M. Castells (1998), Local y global. La gestión de las ciudades en la era de la información, Madrid, Taurus.

Caravaca Barroso, Inmaculada (1998), "Los nuevos espacios emergentes", Revista de Estudios Regionales, enero-abril, pp. 39-80, Universidad de Andalucía.

Carballo G., Carlos y Liliana Pagliettini (1998), "Reestructuración de los sistemas de arroz, ganadería extensiva e integración transfronteriza en el litoral argentino", ponencia presentada en las Jornadas Extraordinarias de Estudios Agrarios "Horacio Giberti", Buenos Aires, UBA/Facultad de Filosofía y Letras.

IDEP-ATE-CTA (1996), “Los nuevos contenidos de las crisis provinciales. Las relaciones fiscales y financieras entre la Nación y las Provincias durante la Convertibilidad", Cuaderno 40, Buenos Aires, Instituto de Estudios sobre Estado y Participación-Asociación de Trabajadores del Estado, 45 págs., ATE.

Íñigo Carrera, N. y M. C. Cotarelo (1997), "Revuelta, motín y huelga en la Argentina actual”, PIMSA Documentos y Comunicaciones, pp. 109-124, Buenos Aires, PIMSA.

_ (1998), "Los llamados 'cortes de ruta", PIMSA Documentos $y$ Comunicaciones, pp. 141-148, Buenos Aires, PIMSA. 
León, Carlos (1994), "Nuevas actividades productivas no tradicionales: alcances y complejidad de su problemática", Realidad Económica, núm. 124, pp. 79-98, Buenos Aires, IADE.

Murmis, Miguel (1998), "El agro argentino, algunos problemas para su análisis", en N. Giarraca y S. Cloquell, Las agriculturas del Mercosur. El papel de los actores sociales, Buenos Aires, La Colmena-ClACSO, pp. 205-248.

Nochteff, Hugo (1996), "La experiencia argentina: ¿desarrollo o sucesión de burbujas", Revista de la CEPAL, núm. 59, agosto, pp. 113-127, Santiago de Chile, CEPAL-UN.

Salvia, A. y M. Panaia (comps.) (1997), La patagonia privatizada. Crisis, cambios estructurales en el sistema regional patagónico y sus impactos en los mercados de trabajo, Buenos Aires, CBC-UBA.

Sartelli, Eduardo (1997), "Mercosur y clase obrera: las raíces de un matrimonio infeliz", Realidad Económica, núm. 146, pp. 38-64, Buenos Aires, IADE.

Senado de la Nación (1998), Informe Argentino sobre Desarrollo Humano 1998, Buenos Aires, Comisión de Ecología y Desarrollo Humano/Honorable Senado de la Nación-Programa de Naciones Unidas para el Desarrollo (PNUD)/Organización de las Naciones Unidas para la Educación, la Ciencia y la Cultura (UNESCO).

Valeiro, Alejandro (1997), “El nuevo algodón: ¿̇on sustentables los cambios recientes del sector algodonero argentino?” y "El algodón en el Mercosur: observaciones sobre su competitividad", Santiago del Estero, comunicaciones del Proyecto Algodón 2000, INTA.

\section{Fuentes periodísticas}

Diario Clarín, Buenos Aires.

Diario La Nación, Buenos Aires.

Revista Apertura (núm. 76, octubre de 1988). 